\title{
e-CATALUNYA, COMUNIDADES DE PRÁCTICA VIRTUALES PARA UNA ADMINISTRACIÓN PÚBLICA MÁS EFICIENTE Y ABIERTA
}

\author{
María-Ribera Sancho, Antonio Cañabate, Albert Botella, Josep Casanovas y \\ Ferran Sabaté
}

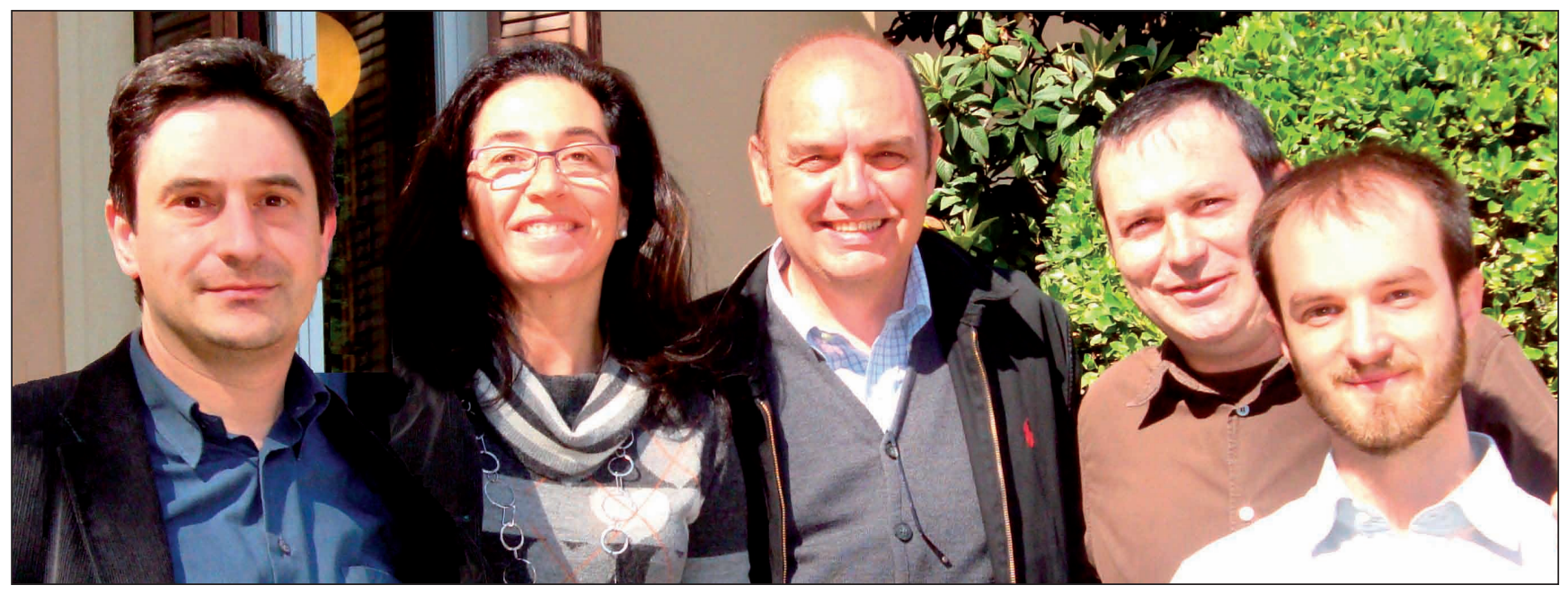

Ferran Sabaté es doctor por la Universitat Politècnica de Catalunya en administración y dirección de empresas, licenciado en informática y posgraduado en formación de formadores. Es profesor del Departamento de Organización de Empresas de la UPC - Barcelona Tech. Colaborador de la Cátedra Telefónica-UPC de análisis de la evolución y tendencias futuras de la sociedad de la información. Ha sido coordinador del área de informática del MBA interuniversitario y coordinador académico del Graduado en auditoría-censura jurada de cuentas (Ccjcc). Su investigación se centra en la aplicación de las tecnologías de la información y comunicación en las empresas, marketing en internet y negocio electrónico, con especial énfasis en la ciencia de los servicios.

Univ. Politècnica de Catalunya, Campus Nord, C5-010A Jordi Girona, 1-3. 08034 Barcelona ferran.sabate@upc.edu

María-Ribera Sancho es profesora titular de la Universitat Politècnica de Catalunya (UPC). Ha trabajado en el campo de la ingeniería del software durante más de 20 años. Fue decana de la Facultat d'Informàtica de la UPC (2004-2010) y vicedecana, jefa de estudios, de la misma facultad (1998-2004). Sus principales áreas de investigación han sido la modelización conceptual de sistemas de información y las metodologías para la ingeniería del software. Su campo de investigación se centra en la ingeniería de los servicios. Es miembro del grupo de investigación MPI (Modelado y procesado de la información) de la UPC. Ha participado en numerosos proyectos para distintas empresas e instituciones.

Univ. Politècnica de Catalunya, Dept. LSI, Campus Nord, C6 Jordi Girona, 1-3. 08034 Barcelona maria.ribera.sancho@upc.edu

Josep Casanovas es Vicerrector de política Universitaria de la Universitat Politècnica de Catalunya - Barcelona Tech y fue decano de la Facultat d'Informàtica de Barcelona. Pertenece al Departamento de Estadística e Investigación Operativa (EIO) y su investigación se enmarca en el grupo LIAM (Laboratory of Information Analysis and Modelling). Es doctor en informática, licenciado en economía e ingeniero industrial. Sus principales áreas de investigación son la simulación, internet y los sistemas de información. Ha participado en diversos proyectos europeos y con otras compañías o instituciones.

Univ. Politècnica de Catalunya, Lab. de Càlcul Facultat d'Informàtica, Campus Nord, B6 Jordi Girona, 1-3. 08034 Barcelona josepk@fib.upc.edu

Antonio Cañabate es profesor de la Universitat Politècnica de Catalunya - Barcelona Tech en el área de organización de empresas. Ha sido vicedecano de la Facultad d'Informàtica (UPC) donde imparte materias de administración de empresas. Ha asesorado a la Generalitat de Catalunya en la planificación del sistema universitario. Ha participado en varios proyectos de investigación en los programas europeos: Tempus, Comett, Phare-ACE en análisis comparativo de la transición económica española y de países de este de Europa y también sobre formación continua. Sus intereses de investigación se centran en el

Artículo recibido el 23-02-11

Aceptación definitiva: 23-03-11 
uso empresarial de las tecnologías de la información y las comunicaciones: e-marketing, comercio electrónico, innovación y productividad, con especial énfasis en los servicios.

Univ. Politècnica de Catalunya, Campus Nord, C5-008 Jordi Girona, 1-3. 08034 Barcelona antonio.canabate@upc.edu

Albert Botella es profesor asociado de la Universitat Politècnica de Catalunya - Barcelona Tech en el Departamento de Ingeniería de Servicios y Sistema de Información (ESSI) donde imparte ingeniería del software y gestión de proyectos. También es consultor sénior en la empresa Altran donde gestiona proyectos sobre redes sociales, movilidad y sistemas de información geográfica (SIG). Ha sido autor de material didáctico y profesor de SIG en la Universitat Oberta de Catalunya. Es profesor del máster en gestión de las tecnologías de la información en la Fundación UPC Tech Talent Center. Sus intereses de investigación incluyen la ciencia de los servicios, redes sociales, gobierno de las TIC y gestión del portfolio de proyectos PPM.

Universitat Politècnica de Catalunya, Omega-108 Jordi Girona, 1-3. 08034 Barcelona albert.botella@upc.edu

\title{
Resumen
}

e-Catalunya es un sistema de información ofrecido por la Generalitat de Catalunya que proporciona un conjunto de herramientas 2.0 para dar soporte a comunidades de práctica virtuales. Facilita la colaboración, la compartición de conocimiento y el cambio hacia una organización más eficiente y abierta. Se describen sus objetivos en el contexto del e-gobierno y de los nuevos patrones de trabajo bajo el paradigma de las comunidades de práctica. Se exponen también las características funcionales y organizativas del sistema, así como su arquitectura. Finalmente se presentan tanto los resultados y beneficios observados como las conclusiones extraídas de la experiencia de 5 años de utilización.

\section{Palabras clave}

Sistemas de gestión del conocimiento, Red social, Trabajo colaborativo, Comunidades de práctica, e-gobierno, Sistemas de información colaborativos, e-Catalunya.

Title: e-Catalunya: virtual communities of practice to promote a more open and efficient public administration

\begin{abstract}
e-Catalunya is an information system promoted by the Government of Catalonia to support Virtual Communities of Practice through a set of web 2.0 tools. It facilitates collaboration, knowledge sharing and the path towards a more open and efficient organization. The information system's goals are reported in the context of both e-government and new working patterns under the Communities of Practice paradigm. Its functional and organizational characteristics as well as its architecture are also described. Finally, observed results and benefits as well as the lessons learned from five years of operation are explained.
\end{abstract}

\section{Keywords}

Knowledge management system, Social networks, Computer supported cooperative work, Communities of practice, eGovernment, Collaborative information systems, e-Catalunya.

Sancho, María-Ribera; Cañabate, Antonio; Botella, Albert; Casanovas, Josep; Sabaté, Ferran. "e-Catalunya, comunidades de práctica virtuales para una administración pública más eficiente y abierta". El profesional de la información, 2011, mayo-junio, v. 20, n. 3, pp. 324-331.

\section{Introducción}

e-Catalunya es un sistema de información ofrecido por la Generalitat de Catalunya y usado con éxito durante los últimos 5 años para dar soporte a las necesidades de colaboración y compartición de conocimiento de empleados públicos, profesionales y ciudadanos, organizados en comunidades de prácticas vinculadas a la prestación de servicios públicos o a proyectos internos o externos que se alinean con las políticas públicas. Es una plataforma enfocada a la colaboración y compartición de conocimiento a través de las fronteras organizativas en un entorno seguro.

Una comunidad de práctica ( $\mathrm{CdP}$ ) es un grupo de personas que comparten su afición por una actividad específica y que interactúan regularmente para aprender y mejorar colectivamente (Lave; Wenger, 1991). Las CdP ayudan a orientar la estrategia, llevar a cabo nuevas líneas de negocio, resolver problemas eficientemente, transferir mejores prácticas, desarrollar habilidades profesionales y a incorporar nuevo talento a la organización. 
Los objetivos de e-Catalunya son obtener una mejora de los servicios públicos prestados y una reducción de costes. Para alcanzarlos, el sistema proporciona a las CdP existentes en distintos departamentos y ámbitos de la administración catalana herramientas para estimular la colaboración, el aprendizaje, la transferencia de buenas prácticas, la toma de decisiones, la implementación de proyectos, etc., garantizando la seguridad y la privacidad.

El gobierno electrónico (e-gobierno), desde su introducción a finales de los 90, hace referencia al uso de internet para ofrecer mejores servicios públicos a los ciudadanos. Sin embargo, es importante entender su potencial transformador de las organizaciones públicas en estados avanzados de desarrollo (Torres; Pina; Acerete, 2005). En el sitio web para la sociedad de la información de la Comisión Europea se define el término e-gobierno como "el uso de herramientas y sistemas construidos mediante tecnologías de la información y las comunicaciones (TIC) para ofrecer mejores servicios públicos a ciudadanos y negocios. [...] Un e-gobierno efectivo también involucra repensar las organizaciones y procesos y cambiar conductas para hacer que los servicios públicos sean más eficientes". Destaca aquí el papel de las TIC como facilitadoras, la mejora de servicios públicos y el potencial de reingeniería.

\section{e-Catalunya es un sistema de informa- ción que da soporte a las necesidades de colaboración de las comunidades de práctica y fomenta su rendimiento}

Se pueden distinguir cuatro tipos de e-gobierno según los agentes que intervienen: gobierno a ciudadanos (G2C), gobierno a negocios (G2B), gobierno a gobierno (G2G) y gobierno a empleados (G2E). Los dos primeros tipos son los más difundidos. Los dos últimos hacen referencia a relaciones entre diferentes departamentos de la administración a diferentes niveles, desde el local hasta el supranacional (G2G), así como los servicios proporcionados a los empleados públicos (G2E).

El modelo G2G difumina las barreras organizativas internas, mientras que los modelos G2C y G2B disipan las externas. Los proyectos $\mathrm{G} 2 \mathrm{E}$ dan soporte a los nuevos requisitos resultantes de los cambiantes patrones de trabajo de los trabajadores del sector público.

El trabajo en organizaciones complejas está cada vez más distribuido. Los trabajadores de la administración, proveedores y ciudadanos colaboran mediante patrones en red no siempre reflejados en las estructuras oficiales (Cross; Parker; Cross, 2004; Grabher; Ibert, 2006). Las tecnologías 2.0 ofre- cen la capacidad de soportar y potenciar las necesidades de los patrones de trabajo actuales (Cohen; Clemens, 2005; McAfee, 2009; Osimo, 2008; Pallot et al., 2006). La creciente exigencia de calidad y eficiencia de los servicios públicos da lugar a una necesidad emergente de colaboración más abierta que cruza fronteras organizativas, pero además requiere tomar en consideración el potencial para obtener valor de la gestión del conocimiento, los procesos de aprendizaje colaborativo y la difusión de mejores prácticas. La mayoría de sistemas que han abordado estos requisitos (Fedorowicz; Gogan; Williams, 2007; Le Dantec; Edwards, 2010) se han planteado para cubrir las necesidades específicas de un grupo para la prestación de un servicio concreto. Por el contrario, e-Catalunya adopta un diseño no especializado capaz de dar soporte a las necesidades de colaboración de cualquier CdP.

El trabajo colaborativo, la red social y la gestión del conocimiento están estrechamente relacionados en los patrones de trabajo actuales en las administraciones públicas. Pueden y deben ser fomentados mediante el uso apropiado de las TIC y tiene sentido abordarlos de forma integral en un mismo sistema, que es la estrategia seguida por e-Catalunya.

Este proyecto se inició a finales de 2004 con la intención de crear una plataforma de e-gobierno innovadora, basada en código abierto, herramientas web 2.0 y capacidades de red social, incorporando desarrollos de grupos de investigación de la Universitat Politècnica de Catalunya - Barcelona Tech (UPC). Para comprender el punto de partida es necesario recordar que, por entonces, las redes sociales no tenían ni el desarrollo ni la difusión popular de que gozan hoy día. Facebook acababa de nacer en Stanford hacía unos meses. El primer piloto data de 2005 y el servicio se empieza a ofrecer en abril de 2006. Desde entonces ha ido creciendo y evolucionando tanto en funciones como en su nivel de utilización. La figura 1 muestra la evolución desde sus orígenes.

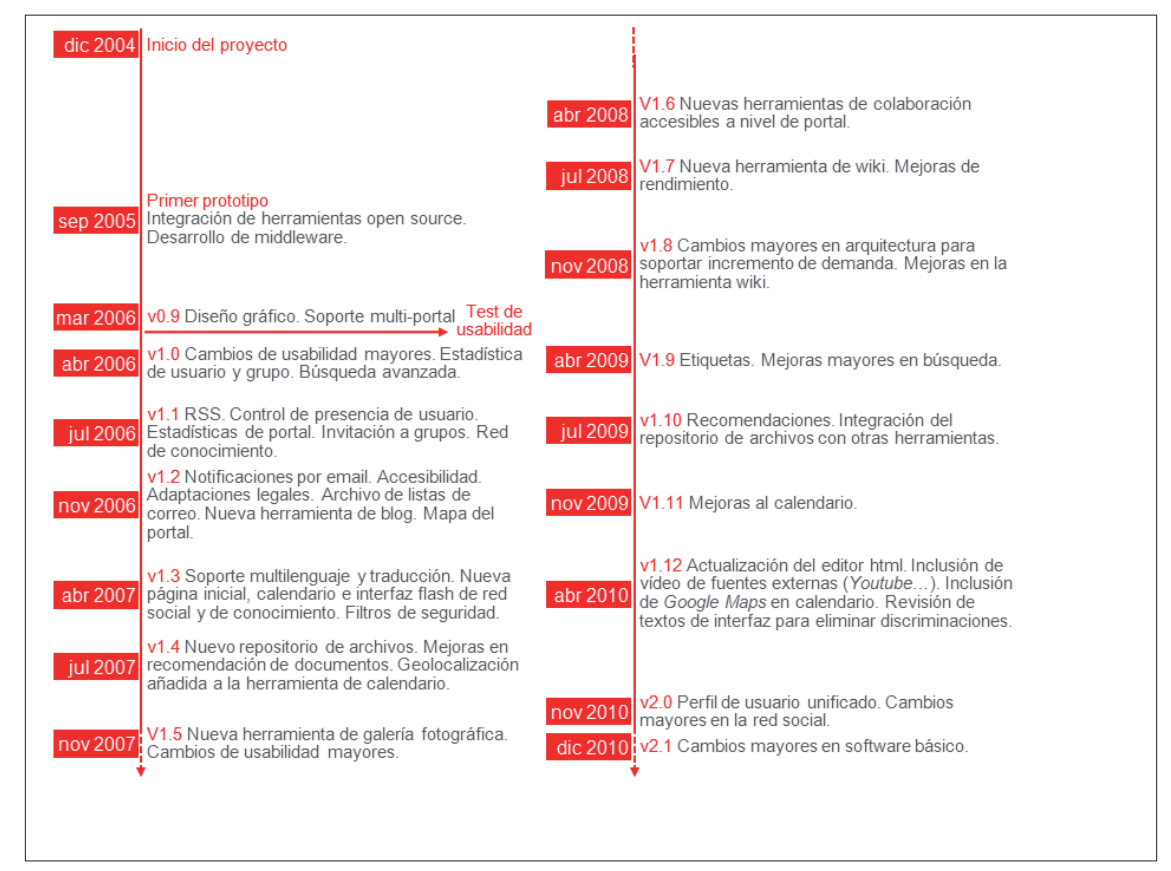

Figura 1. Evolución del proyecto e-Catalunya 


\section{El sistema e-Catalunya}

e-Catalunya funciona en una plataforma que ofrece un conjunto de herramientas web 2.0 y se diferencia de otras alternativas en la "nube" por su énfasis en la accesibilidad, usabilidad y privacidad, lo cual limita decisivamente el conjunto de soluciones posibles. Deben respetarse fuertes requisitos de protección de datos, seguridad y privacidad de la información. Los datos y perfiles de usuario permanecen seguros bajo la tutela de estructuras gubernamentales. La accesibilidad debe estar certificada con acuerdo a los requisitos de la legislación española.

Como sistema de información que es e-Catalunya afronta el reto de mejorar la organización de la Generalitat de Catalunya, una administración con una estructura compleja y fragmentada. En consecuencia, el diseño del sistema ha involucrado a funcionarios públicos (sus destinatarios en origen), profesionales independientes y ciudadanos que colaboran en las diferentes CdP.

\section{e-Catalunya ofrece varias aplicaciones} web 2.0 y se diferencia de otras alternativas por su énfasis en la accesibilidad, usabilidad y privacidad

\section{Funciones}

Se organizan en cuatro categorías:

1) descubrir y comunicarse con profesionales de su campo $\mathrm{u}$ otras personas con quienes los usuarios comparten intereses;

2) acceso al contenido y conocimiento generado por éstos de un modo seguro y centralizado;

3) contribuir al conocimiento general de la organización involucrándose en iniciativas de participación pública; y

4) identificar tendencias, alcanzar consensos, iniciar y consolidar proyectos.

\begin{tabular}{|l|l|}
\hline \multicolumn{1}{|c|}{$\begin{array}{c}\text { Aplicaciones } \\
\text { de gestión }\end{array}$} & \multicolumn{1}{|c|}{ Aplicaciones de colaboración } \\
\hline $\begin{array}{l}\text { Almacenamiento de } \\
\text { ficheros organizados en } \\
\text { carpetas jerárquicas }\end{array}$ & Wikis, blogs, foros, calendarios \\
\hline Listas de distribución & $\begin{array}{l}\text { Redes sociales y mapas de redes de } \\
\text { conocimiento } \\
\text { (representación visual de las redes } \\
\text { relacionales y participación en ámbitos) }\end{array}$ \\
\hline Álbumes de fotos & $\begin{array}{l}\text { Procesos participativos } \\
\text { (cuestionarios, encuestas y herramientas } \\
\text { de explotación) }\end{array}$ \\
\hline RSS y alarmas & Nubes de etiquetas \\
\hline $\begin{array}{l}\text { Estadísticas de uso a } \\
\text { nivel de plataforma, } \\
\text { portal, grupo y usuario }\end{array}$ & \\
\hline
\end{tabular}

Tabla 1. Aplicaciones para usuarios y administradores

\section{Estructura organizativa}

e-Catalunya se organiza en portales, normalmente asociados a un área de la organización -un departamento o una institución pública-. Como ejemplos de portales que han tenido más éxito debemos citar el de Justicia (Pérez-Montoro; Martínez, 2007), el de Salud y el del Instituto de la Mujer. Cada uno puede contener una jerarquía de grupos/subgrupos con un número ilimitado de niveles. Los usuarios pueden contribuir y tener acceso a distintos grupos.

Cada portal es gestionado por un administrador que controla los permisos sobre los contenidos y las herramientas disponibles, así como la creación de grupos y usuarios. Actúa como promotor creando grupos de personas competentes en su área de interés, independientemente de sus dependencias organizativas, para facilitar los contactos entre ellos.

El grupo se considera el facilitador de una CdP y es totalmente independiente en el seno de la plataforma, pudiendo tener su propio administrador. Éste define las herramientas disponibles específicamente para su grupo. Por ejemplo, cada grupo puede tener su propio foro, blog y almacén de ficheros.

En las versiones anteriores el perfil de un usuario era distinto para cada portal en que participaba (Casanovas et al., 2009). A partir de la versión 2.0 se han mejorado las funciones de red social y los perfiles de usuario son únicos, al tratarse de una red social global. Así se facilita que un usuario de una CdP pueda descubrir otras comunidades de su interés.

A partir de la versión 2.0 se han mejorado las funciones de red social y los perfiles de usuario son únicos

\section{Promoción, apoyo y formación}

La implantación de este tipo de sistema de información requiere formación y apoyo a los usuarios, no sólo en el uso de la propia plataforma, sino también sobre cómo aprovecharla para mejorar la colaboración y la eficiencia en sus tareas.

Cuando una CdP es autorizada a unirse a e-Catalunya, si ya existe un portal relacionado con su ámbito su administrador crea un nuevo grupo; en caso contrario, el administrador del sistema crea un nuevo portal. Los promotores de la plataforma proporcionan asesoramiento y formación a los futuros administradores para facilitar la puesta en marcha, así como apoyo técnico y funcional mediante correo electrónico y teléfono.

El gobierno de Catalunya organiza eventos y actividades de formación y apoyo para promover el uso del sistema, detectar nuevas CdP y facilitar el contacto presencial entre personas que comparten objetivos.

\section{Arquitectura}

\section{Arquitectura lógica}

El sistema de información se ajusta a la arquitectura de 3 capas modelo-vista-controlador (Gamma et al., 1994). La capa de presentación se implementa mediante portlets, pero 
también se utilizan servlets y servicios web. La lógica de negocio y la integración de herramientas es implementada en una capa intermedia que sigue el patrón fachada de ingeniería del software para ofrecer un único punto de acceso a los servicios. La integración se lleva a cabo mediante el patrón adaptador para cubrir las particularidades de cada herramienta. La comunicación con esta capa de integración se realiza mediante el patrón observador para independizar la lógica de negocio de la integración. Por último, la capa de acceso a datos se gestiona mediante Hibernate para el acceso a la base de datos relacional mySQL. Todos estos componentes se unen con Exoplatform que proporciona un contenedor de portlets para la presentación, una capa de servicios y también la capa de acceso a datos.

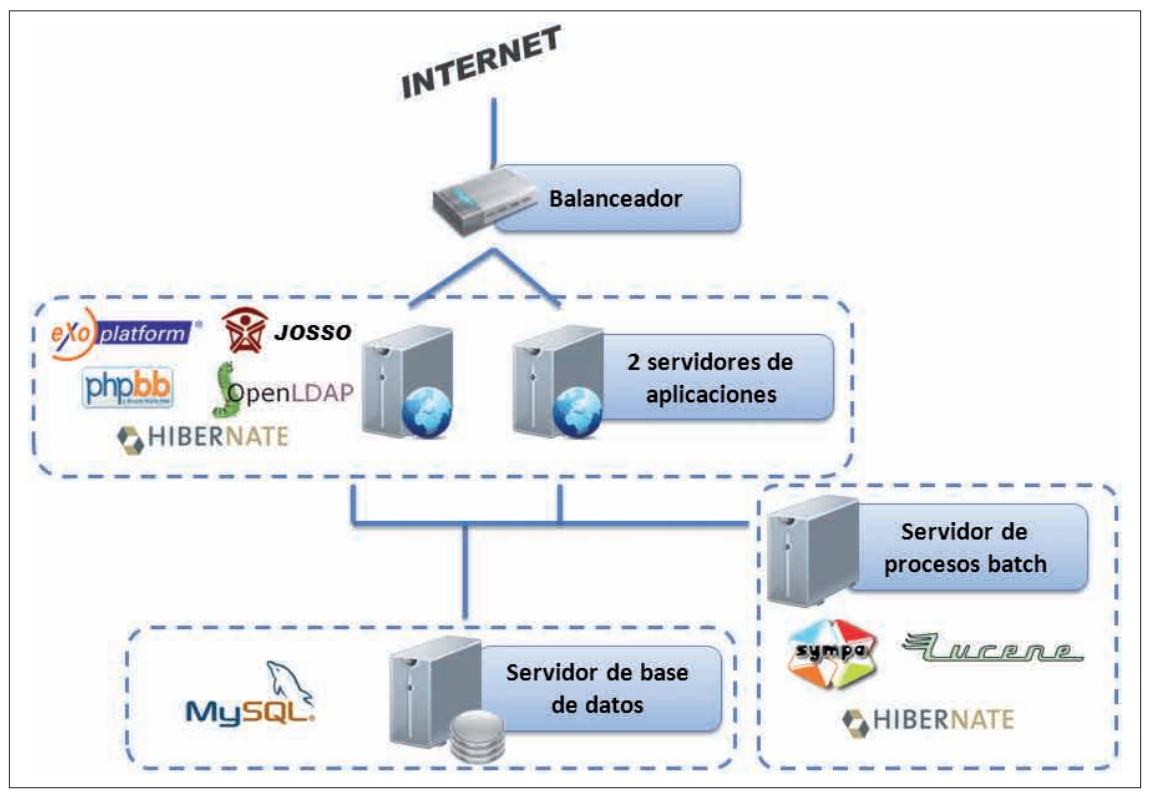

Figura 2. Arquitectura física

Se usa el sistema operativo Linux (compatible con Suse Linux y RedHat), el servidor de aplicaciones Apache/Tomcat y el entorno java y php. Esta arquitectura aporta garantías para la ejecución a gran escala y alta disponibilidad. Se ha llevado a cabo integrando herramientas de código libre (tabla 2) enlazadas mediante componentes programados a medida. Sin embargo, algunas piezas, por ejemplo la wiki, se han diseñado a medida tras descartar componentes de código libre como xwiki por no cubrir completamente los requisitos de usuario.

La arquitectura aporta garantías para la ejecución a gran escala y alta disponibilidad. Integra herramientas de código libre enlazadas mediante componentes programados a medida

ciendo. La figura 2 muestra la arquitectura física actual y dónde se ejecuta cada una de las herramientas de código libre.

Los servidores de aplicaciones se han virtualizado para mejorar la escalabilidad en caso del rápido aumento de usuarios. El servidor de procesos por lotes (batch) realiza la indexación, los cálculos de indicadores del cuadro de mando, la generación de alertas de noticias y los cálculos de contactos de la red social. Es un servidor dedicado para minimizar el impacto de la ejecución de estos pesados procesos sobre el rendimiento y disponibilidad del sistema.

La última versión de e-Catalunya desplegada en marzo de 2011 incluye: 1) toda la navegación bajo el protocolo https; 2) actualización a las últimas versiones del sistema operativo RedHat y del servidor de aplicaciones Apache/Tomcat y 3) actualización a la última versión de java y php.

Se están evaluando componentes para incorporar mensajería instantánea sobre protocolo Jabber.

\section{Resultados}

Tras 5 años de funcionamiento de e-Catalunya y según muestra la figura 3, la difusión ha alcanzado en 2010 un to-

La infraestructura física de soporte hardware ha ido evolucionando a medida que el número de usuarios ha ido cre-

\begin{tabular}{|l|l|}
\hline Herramienta & \multicolumn{1}{c|}{ Descripción } \\
\hline MySQL & Sistema gestor de bases de datos. \\
\hline Exoplatform & $\begin{array}{l}\text { Herramienta para la gestión de contenidos web que implementa la especificación JSR 168 para la gestión de portlets. Es la base } \\
\text { integradora para la construcción y navegación de e-Catalunya. }\end{array}$ \\
\hline PhPBB & Sistema de foros desarrollado en php. \\
\hline Lucene & $\begin{array}{l}\text { Librería escrita en java para la indexación y búsqueda de texto con la que se implementa la búsqueda de contenidos así como la } \\
\text { generación de alertas y novedades de interés para el usuario. }\end{array}$ \\
\hline Josso & $\begin{array}{l}\text { Solución de autenticación única (single sign-on) que permite mantener la sesión de usuario durante toda la navegación por la } \\
\text { plataforma. }\end{array}$ \\
\hline OpenLdap & Implementación de LDAP (light weight directory access protocol) que almacena los datos de los usuarios y facilita la autenticación. \\
\hline FCKEditor & Editor de texto enriquecido para web que incluye imágenes y elementos multimedia. \\
\hline Sympa & Gestión de listas de correo. \\
\hline
\end{tabular}

Tabla 2. Algunas de las herramientas de código libre integradas en e-Catalunya 


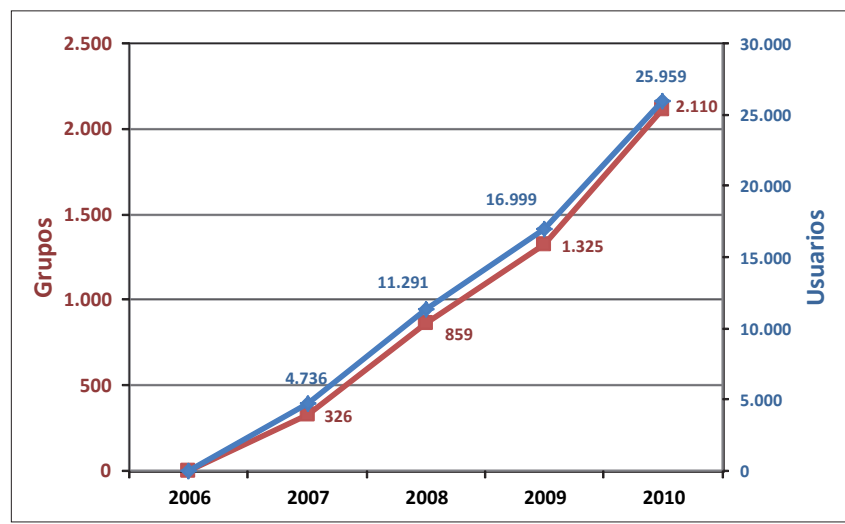

Figura 3. Número de grupos y usuarios en e-Catalunya

tal de 25.959 usuarios registrados organizados en más de 2.110 grupos de trabajo, ocurriendo el mayor incremento de usuarios y grupos en el último año.

La creación de portales ha sido intensa desde un inicio hasta alcanzar y superar los 70 . Su crecimiento se estabilizó en el 2010 (figura 4) en tanto que los portales existentes ya cubren gran parte de ámbitos de la administración catalana. El crecimiento ocurre en nuevos grupos y usuarios dentro de cada portal. En cuanto al uso, se reciben entre 30.000 y 40.000 visitas mensuales por parte de los miembros de las diferentes CdP integradas en el sistema.

De entre los portales más numerosos destacar, en primer lugar, el de Justicia con 3.545 usuarios que fue el piloto de la plataforma. Se trata posiblemente del caso más exitoso en cuanto a potenciar las ventajas de las CdP y de la gestión del conocimiento. Su amplia difusión nacional e internacional

\section{En 2010 se tenían 25.959 usuarios regis- trados organizados en 2.110 grupos de trabajo}

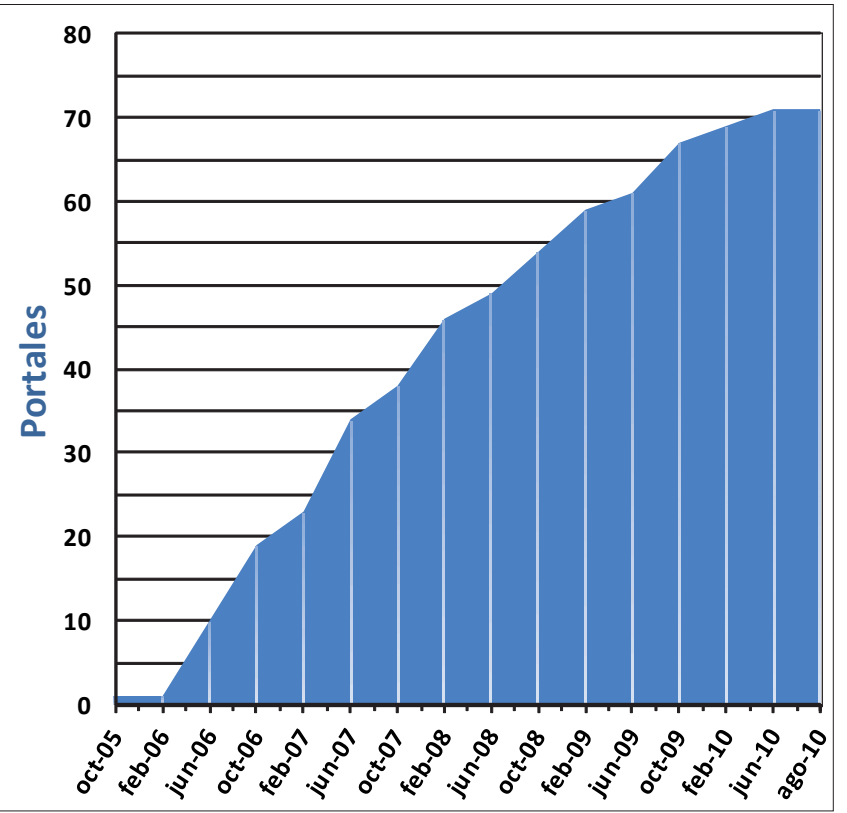

Figura 4. Número de portales en e-Catalunya ha permitido, a su vez, la difusión de la propia plataforma. Sobre este portal se implementó el proyecto del Departament de Justícia de la Generalitat de Catalunya con el fin de capturar y gestionar el conocimiento que hasta ese momento estaba disperso geográficamente y aislado en las mentes de los profesionales del área. Inicialmente el proyecto se centró en los asistentes sociales y cuatro meses después, tras haber analizado los primeros resultados, se amplió progresivamente a otras comunidades, tales como jueces, psicólogos, etc. Con este proyecto el Departament de Justícia consiguió incrementar la creatividad y mejorar las prácticas de trabajo del sector en base a una mejor gestión del conocimiento. Por otra parte, la implementación del portal aportó las primeras experiencias prácticas en elementos clave de la plataforma, tales como el rol del moderador y los métodos de trabajo de las comunidades (Pérez-Montoro; Martínez, 2007).

En segundo lugar, el portal de Educación, que cuenta con 5.477 usuarios y 359 grupos. En sus CdP participan inspectores, profesores y empleados del gobierno. Así por ejemplo, los inspectores comparten planes entre ellos, los profesores comparten estrategias docentes y los empleados del gobierno facilitan recursos de aprendizaje.

En tercer lugar, el portal público Barrios con proyectos reúne 455 miembros en un único grupo cuyo objetivo común es crear un espacio virtual de encuentro, intercambio y de información para quienes trabajan en proyectos de mejora integral de barrios.

Por último, Catalunya exterior consta de 350 usuarios en 8 grupos $^{1}$. Se autodefine como un portal vinculado a la Secretaría de Asuntos Exteriores cuyo objetivo es crear una red social entre personas y entidades catalanas localizadas fuera de Catalunya. El sistema posibilita la comunicación entre distintos colectivos y la elaboración de proyectos comunes.

\section{El portal de Justicia es posiblemente el caso más exitoso en cuanto a potenciar las ventajas de las $\mathrm{CdP}$ y de la gestión del conocimiento}

Según análisis del proveedor del servicio, la Generalitat de Catalunya, el soporte a la colaboración y a la gestión de conocimiento que el sistema e-Catalunya aporta a sus CdP ha mejorado su eficiencia organizativa sobre varios aspectos. El tráfico de datos se ha reducido como consecuencia de la disponibilidad de datos compartidos y el soporte de interacciones multipunto evita la replicación innecesaria de datos y optimiza el tráfico.

El sistema disminuye la necesidad de celebrar reuniones de trabajo presenciales. El gobierno catalán estima una reducción entre un 25 y un $50 \%$ en desplazamientos por este motivo, con recorridos medios estimados de $120 \mathrm{~km}$.

La utilización de procedimientos estándar basados en el trabajo colaborativo reduce entre un 40 y un $60 \%$ la duración de la fase de definición de proyectos que en organizaciones complejas es de entre 4 y 6 meses en promedio. 
e-Catalunya impulsa el aprendizaje colaborativo y la transferencia de buenas prácticas de manera natural en el seno de cada CdP. El coste de la formación en estos casos se reduce drásticamente respecto a la formación más tradicional, llegando a alcanzar el $100 \%$ o alrededor del $50 \%$ en los casos en que el líder de la comunidad es remunerado por su labor de soporte al aprendizaje.

Tales mejoras son la razón de ser del sistema e-Catalunya, un servicio que permite al gobierno catalán ser más eficiente y reducir costes facilitando el trabajo colaborativo y la gestión del conocimiento, siendo la inversión necesaria muy inferior a los beneficios (Graells, 2010).

e-Catalunya impulsa nuevos patrones de trabajo colaborativo en una administración más abierta, mejorando el rendimiento de sus CdP

\section{Conclusiones}

La creciente exigencia de calidad y eficiencia de los servicios públicos da lugar a una necesidad emergente de colaboración entre los miembros de las $\mathrm{CdP}$ relacionadas con los servicios prestados. Frente a un modelo unidireccional de prestación de servicios públicos, las $\mathrm{CdP}$ reflejan la realidad de una colaboración más abierta entre departamentos de la administración, profesionales externos y ciudadanos que comparten sus prácticas para aprender colectivamente y colaboran en la mejora de los servicios públicos.

e-Catalunya es un sistema de información proporcionado por la Generalitat de Catalunya capaz de dar soporte a las necesidades de colaboración de cualquier CdP y fomentar su rendimiento mediante el uso apropiado del conjunto de herramientas 2.0 que ofrece. Así lo demuestra el éxito de adopción y uso en sus 5 años de vida, su crecimiento en número de usuarios y grupos y la existencia de portales para gran parte de los ámbitos clave de la administración.

La experiencia de más de 5 años de operación de e-Catalunya arroja algunas enseñanzas (Continente, 2010). Las herramientas del sistema son facilitadoras, pero para sacar partido de las posibilidades que ofrecen, es necesaria la concurrencia de factores no tecnológicos. Así, los miembros de las CdP necesitan instrucción y soporte en estrategias de aprendizaje colaborativo, así como en aspectos de propiedad intelectual, confidencialidad y privacidad. Moderadores y administradores son clave para el éxito de una CdP. Identificar y motivar a los líderes de las CdP para que actúen de catalizadores de dinámicas de grupo, promuevan la colaboración, ayuden a nuevos miembros a superar miedos y desconfianzas iniciales hacia las herramientas 2.0 como blogs o forums, es decisivo. La motivación a participar debe ser estimulada con incentivos, especialmente con el reconocimiento a la experiencia y conocimiento aportados. Los encuentros presenciales ayudan a establecer relaciones virtuales. Cuando se constituye una nueva $\mathrm{CdP}$, un primer contacto presencial es útil para definir esquemas de colaboración estables y eficientes en la posterior relación virtual (Pérez-Montoro; Martínez, 2008).
Los beneficios del sistema en términos de eficiencia y ahorros de costes han sido constatados. E-Catalunya demuestra también su capacidad para impulsar nuevos patrones de trabajo colaborativo en una administración más abierta mejorando el rendimiento de sus comunidades de práctica.

\section{Nota}

1. Datos actualizados en enero de 2011.

\section{Agradecimientos}

Este trabajo ha sido posible gracias a la colaboración de la Generalitat de Catalunya y la Universitat Politècnica de Catalunya-Barcelona Tech.

\section{Bibliografía}

Casanovas, Josep; Continente, Marta; Fernández, Marta; Golobart, Daniel; Graells, Jordi; Martín, Rosa M.; Ponce, Lucas; Puente, Héctor. "E-Catalunya experience report (July 2009)". En: Incos'09. Proc of the 2009 intl conf on intelligent networking and collaborative systems, pp. 67-73. ISBN 9780769538587. DOI:10.1109/INCOS.2009.60

Cohen, Tracy; Clemens, Ben. "Social networks for creative collaboration". En: The $5^{\text {th }}$ conf on creativity \& cognition, 2005, pp. 252-255. ISBN 1595930256. DOI:10.1145/10562 24.1056265 .

Continente, Marta. "e-Catalunya: boosting eGov innovation by communities of practice". Epractice.eu, 2 Oct 2009.

http://epractice.eu/en/cases/ecatalunya

Cross, Robert; Parker, Andrew; Cross, Rob. The hidden power of social networks: understanding how work really gets done in organizations. Harvard Business Press, 2004, ISBN 1591392705.

Fedorowicz, Jane; Gogan, Janis L.; Williams, Christine B. "A collaborative network for first responders: lessons from the CapWIN case". Government information quarterly, 2007, October, v. 24, n. 4, pp. 785-807.

Gamma, Erich; Helm, Richard; Johnson, Ralph; Vlissides, John M. Design patterns: elements of reusable object-oriented software. Addison-Wesley Professional, 1994. ISBN 0201633612.

Grabher, Gernot; Ibert, Oliver. "Bad company? The ambiguity of personal knowledge networks". Journal of economic geography, Jun. 2006, v. 6, n. 3, pp. 251-271.

Graells, Jordi. Per què és més productiu treballar col-laborativament a l'Administració? Desembre 2009.

http://www.slideshare.net/jordigraells/productivitat-copadministraci-cat

Lave, Jean; Wenger, Etienne. Situated learning: legitimate peripheral participation. Cambridge University Press, 1991. ISBN 0521423740.

Le Dantec, Christopher A.; Edwards, W. Keith. "Across boundaries of influence and accountability: the multiple scales of public sector information systems". En: The $28^{\text {th }}$ intl conf on human factors in computing systems, 2010, pp. 113-122. 
http://www.ledantec.net/wp-content/uploads/2010/02/ pap0791-ledantec.pdf

McAfee, Andrew. Enterprise 2.0: new collaborative tools for your organization's toughest challenges. Harvard Business School Press, 2009. ISBN 1422125874.

Osimo, David. Web 2.0 in government: why and how? Luxembourg: Office for Official Publications of the European Communities, 2008, ISSN 1018-5593.

ftp://ftp.jrc.es/pub/EURdoc/JRC45269.pdf

Pallot, Marc; Ruland, Rudolf; Traykov, Stanislav; Kristensen, Kjetil. "Integrating shared workspace, wiki and blog technologies to support interpersonal knowledge connection". En: The $12^{\text {th }}$ intl conf on concurrent enterprising, ICE, 2006.
Pérez-Montoro, Mario; Martínez, Jesús. "Enabling knowledge creation in judicial environments: the case of Catalonia's Public Administration". En: Martin, B., Remenyi, D. (eds). ECKM 2007. $8^{\text {th }}$ European conf on knowledge management. London: Academic Conferences Limited Reading, 2007, pp. 766-773. ISBN 9781905305537.

Pérez-Montoro, Mario; Martínez, Jesús. "Success factors of communities of practice in Public Administration: the case of Catalonia's Government". En: The $5^{\text {th }}$ intl conf on intellectual capital: Icickm 2008, 2008, pp. 407.

Torres, Lourdes; Pina, Vicente; Acerete, Basilio. "E-government developments on delivering public services among EU cities". Government information quarterly, 2005, v. 22, n. 2, pp. 217-238.

\section{部IS IWETEL \\ Foro para profesionales de http://www.rediris.es/list/info/iwetel.html bibliotecas y documentación}

\section{IweTel, foro de información y debate de la biblioteconomía y la documentación}

Fundada por Tomàs Baiget en 1993, IweTel es la lista pionera en español de los profesionales de las bibliotecas, documentación, bases de datos y sistemas de información en general.

Al principio se alojó en Sarenet y en 1998 pasó a RedIRIS. Posteriormente se han ido creado otras listas más especializadas como Arxiforum (archivos), Bib-Med (información bio-médica), Bescolar (bibliotecas escolares), Incyt (indicadores científicos), etc., pero IweTel, con más de 5.000 miembros, es la lista de referencia, el medio de comunicación básico y central para los profesionales de la información.

En la lista se cumple la conocida regla del $80 / 20$ (el $80 \%$ de los mensajes los genera el $20 \%$ de los inscritos), o su reciente reformulación a 90, 9, 1\%: el 90\% de los inscritos son pasivos, casi nunca envían nada, el 9\% (unos 360) participa alguna vez, y existe un 1\% (50 personas) que genera la mayoría de mensajes.

Con el aumento de inscritos y el número de mensajes (algunas semanas se distribuyen más de 100) fue necesario hacer la lista moderada, y en ello estamos los 4 firmantes, intentando aplicar nuestro sentido común para decidir cuáles se aprueban y cuáles no, y evitando los mensajes repetidos. Rechazamos alrededor de un 15-20\%, lo cual a veces provoca quejas de sus autores, y para dirimir las dudas se creó un Consejo Asesor formado por veteranos de la lista, a quienes los moderadores pedimos consejo.

La lista cumple los dos objetivos básicos típicos: tablón de anuncios (conferencias, cursos, publicaciones, noticias) y foro de debates. Además se usa como sistema abierto de evaluación por pares (open peer review) de las notas que los miembros del think tank ThinkEPI envían periódicamente a la lista para su pública crítica y discusión. Esas notas y los principales mensajes que generan se publican cada año re-editados en el Anuario ThinkEPI de la editorial EPI.

Con los cambios tecnológicos habidos a lo largo de estos años y, más recientemente, con las nuevas plataformas web 2.0, se ha planteado muchas veces si las listas de correo se han hecho "obsoletas". La verdad es que pensamos que una lista sigue siendo el medio ideal de comunicación de una comunidad profesional: rápida, limpia, discreta y eficaz, lejos de la faramalla de las redes sociales, también muy interesantes y útiles pero para otras cosas.

Más información e inscripciones:

http://www.rediris.es/list/info/iwetel.html

Javier Leiva-Aguilera (Catorze.com), Paco López-Hernández (Universidad Carlos III de Madrid), Isabel Olea (Universidad de León) y Tomàs Baiget (El profesional de la información). 